\title{
Germline deletion of FAK-related non-kinase delays post-natal cardiomyocyte mitotic arrest
}

\author{
Thomas J. O'Neill'1,2, Christopher P. Mack ${ }^{1,3}$, and Joan M. Taylor ${ }^{1,3, \#}$ \\ ${ }^{1}$ Department of Pathology, University of North Carolina, Chapel Hill, NC 27599, USA \\ ${ }^{2}$ Department of Medicine, University of North Carolina, Chapel Hill, NC 27599, USA \\ ${ }^{3}$ McAllister Heart Institute, University of North Carolina, Chapel Hill, NC 27599, USA
}

\section{Abstract}

The cardiomyocyte phenotypic switch from a proliferative to terminally differentiated state impacts normal heart development and pathologic myocardial remodeling, yet the signaling mechanisms that regulate this vital process are incompletely understood. Studies from our lab and others indicate that focal adhesion kinase (FAK) is a critical regulator of cardiac growth and remodeling and we found that expression of the endogenous FAK inhibitor, FAK-related non kinase (FRNK) coincided with postnatal cardiomyocyte arrest. Mis-expression of FRNK in the embryonic heart led to pre-term lethality associated with reduced cardiomyocyte proliferation and led us to speculate that the postnatal FRNK surge might be required to promote quiescence in this growth promoting environment. Herein, we provide strong evidence that endogenous FRNK contributes to post-mitotic arrest. Depletion of FRNK promoted DNA synthesis in post-natal day (P) 10 hearts accompanied by a transient increase in DNA content and multi-nucleation by P14, indicative of DNA replication without cell division. Interestingly, a reduction in tri- and tetranucleated cardiomyocytes, concomitant with an increase in bi-nucleated cells by P21, indicated the possibility that FRNK-depleted cardiomyocytes underwent eventual cytokinesis. In support of this conclusion, Aurora B-labeled central spindles (a hallmark of cytokinesis) were observed in tetra-nucleated P20 FRNK ${ }^{-/}$but not wt cardiomyocytes, while no evidence of apoptosis was observed. Moreover, hearts from FRNK null mice developed ventricular enlargement that persisted until young adulthood which resulted from myocyte expansion rather than myocyte hypertrophy or interstitial growth. These data indicate that endogenous FRNK serves an important role in limiting DNA synthesis and regulating the un-coupling between DNA synthesis and cytokinesis in the post-natal myocardium.

\section{Keywords}

cardiomyocyte; polyploidy; Focal adhesion kinase; FAK-related non-kinase; cell cycle

\footnotetext{
(C) 2012 Elsevier Ltd. All rights reserved.

${ }^{\#}$ To whom correspondence should be addressed: Joan M. Taylor, Ph.D., Department of Pathology and Lab Medicine, 501 BrinkhousBullitt Building. CB 7525, University of North Carolina, tel. \# : (919) 843-5512, fax \#: (919) 966-6718, jmt3x@ med.unc.edu. DISCLOSURES: None

Publisher's Disclaimer: This is a PDF file of an unedited manuscript that has been accepted for publication. As a service to our customers we are providing this early version of the manuscript. The manuscript will undergo copyediting, typesetting, and review of the resulting proof before it is published in its final citable form. Please note that during the production process errors may be discovered which could affect the content, and all legal disclaimers that apply to the journal pertain.
} 


\section{INTRODUCTION}

While recent evidence suggests that a small subset of mammalian cardiomyocytes retain the ability to enter the cell cycle throughout their lifespan[1], it is clear that most adult cardiomyocytes are terminally differentiated and not capable of cell division[2]. This is one of the primary reasons that myocardial infarction in adult patients results in permanent loss of muscle mass even after the restoration of blood flow[3]. However, while adult hearts are notorious for their lack of regenerative capacity, recent studies revealed that injury to hearts that contain cycling cardiomyocytes (such as zebrafish or newborn mouse hearts) led to remarkable myocardial regeneration through enhanced myocyte proliferation [4, 5]. These findings highlight the importance of a clear understanding of myocyte cell cycle regulation, as targeting cell cycle re-entry could lead to fundamental advancements in the treatment of cardiac diseases associated with reduced myocyte viability.

Cardiomyocytes are highly proliferative during heart formation in mid-gestation, but their proliferation slows during the late antenatal period and, within the first 2 weeks following birth, cardiomyocytes largely lose the capacity to undergo coupled karyo- and cytokinesis[6]. Thus the elevated DNA synthesis that occurs in the neonatal heart, particularly in the left ventricle following postnatal circulatory pattern switching, results in bi-nucleation and subsequent cell cycle arrest. In the mouse, this so-called multi-nucleation phase is completed around 2 weeks after birth. During this same time period cardiomyocytes will also transition into a hypertrophic growth phase which is responsible for most of the juvenile cardiac growth[2]. While several studies have defined the positive and negative cell cycle regulators that influence myocyte proliferation during heart formation[7], less is known about the signals that regulate the neonatal transition to permanent cell cycle withdrawal.

Several studies indicate that the cardiomyocyte growth state in the developing heart correlates with regulated shifts in the expression of extracellular matrix and integrin receptors and the ability of these matrices to support myocyte growth. Indeed, the growth promoting matrix, fibronectin (FN) is highly expressed in the developing myocardium, while the growth limiting matrix, collagen I, becomes much more abundant after birth[8]. Moreover, it is clear from prior studies from our laboratory and others that FAK, a critical mediator of FN-binding integrin receptors, functions to facilitate cardiomyocyte proliferation during heart formation $[9,10]$.

FAK activity can be regulated by the expression of its endogenous inhibitor, FRNK.[11, 12] The FRNK protein is comprised of the carboxyl terminus of FAK (which lacks the catalytic domain), and FRNK likely functions to limit FAK activity either by direct interaction (since FAK undergoes dimerization-induced activation)[13] or by competing for FAK's binding partners[12, 14]. Interestingly, FRNK transcription results from the utilization of an alternative start site within the FAK gene that is driven by a distinct promoter embedded within FAK intronic sequences. We previously showed that whereas FAK expression was relatively constant during heart development, FRNK was transiently expressed in the heart with peak levels occurring just prior to neonatal cell cycle withdrawal[15]. Importantly, we reported that conditional mis-expression of FRNK in the embryonic heart led to peri-natal lethality associated with impaired cardiomyocyte proliferation and ventricular noncompaction. Our findings lead us to propose that post-natal expression of cardiac FRNK might dampen FAK-dependent cardiomyocyte cell cycle progression and facilitate cell cycle exit. Herein, we report that hearts from FRNK null mice exhibited increased DNA synthesis and hyperplastic growth into young adulthood, indicating that endogenous FRNK expression is a major contributor to post-natal cardiomyocyte arrest. 


\section{METHODS}

\section{Antibodies}

The FAK, FRNK, phospho-FAK and phospho-Histone H3 antibodies were purchased from Millipore. Antibodies against Tropomyosin were obtained from Sigma while Aurora B and Histone $\mathrm{H} 3$ antibodies were purchased from AbCam. The cardiac troponin I antibody was purchased from Thermo, and the N-Cadherin, and GAPDH antibodies were obtained from Santa Cruz. All Alexa-fluor labeled secondary antibodies as well as Alexa-647 labeled WGA lectin and Alexa-555 labeled Strepavidin were obtained from Invitrogen. DAPI and propidium iodine was obtained from Sigma.

\section{Mouse Models}

The FRNK ${ }^{-1-}$ mice were obtained from Dr. Tom Parsons and have been previously described[16]. Mice were genotyped by PCR using previously described primers and DNA isolated from tail snips.

\section{BrdU Injections}

Cardiac cell proliferation was determined by BrdU labeling which was achieved by administering $40 \mathrm{mg} / \mathrm{Kg}$ of BrdU (Sigma) by intraperitoneal injections. The hearts were harvested 3 days later, fixed, and embedded in paraffin. BrdU incorporation was detected by using a commercially available kit (Invitrogen) combined with Alexa-555 labeled strepavidin. Cell proliferation was quantified by scoring the number of BrdU-positive nuclei per unit area using NIH Image $\mathbf{J}$ software.

\section{Myocyte cell isolation and flow cytometry}

Ventricular cardiomyocytes were isolated from neonatal mice by trypsin and collagenase (Worthington) digestion and purified as described previously[15]. After digestion into a single cell suspension, cells were fixed by dropwise addition of cold $70 \%$ ethanol. After fixation they were resuspended in Hank's buffered salt solution and stained with propidium iodide (1:10,000 dilution). In the case of antibody staining with $\mathrm{N}$-Cadherin the cells were not fixed but were incubated with primary antibody, followed by Alexa 488 labeled secondary antibody then and washed and permeabilized with Triton- $\mathrm{X}$ before propidium labeling and analysis. All cells were run on an Accuri C6 flow cytometer and analyzed using Accuri software. For quantification of cardiomyocyte number, the entire population was counted using the aforementioned method with cardiomyocytes identified by forward and side scatter properties. For cell staining, after digestion cells were preplated on plastic for 1 hour then were plated on glass chamber slides pretreated with fibronectin or laminin for 224 hours before fixation and staining.

\section{Histological Analysis}

Paraformaldehyde fixed, paraffin-embedded hearts were sectioned into $10 \mu \mathrm{m}$ sections, which were permeabilized with phosphate buffered saline containing $2 \%$ bovine albumin with $0.3 \%$ Triton-X and/or $0.1 \%$ Saponin (Sigma) and then incubated indicated antibodies overnight at 1:50-1:200. Secondary antibodies at 1:500-1:2000 dilution were incubated at room temperature for 1 hour after which slides were washes and sealed with Fluoromount (Southern Biotech). Cell area measurements were conducted on Alexa-647- WGA Lectin stained cross sections of the compact zone of the left ventricle using Image $\mathrm{J}$ software.

\section{Western Blotting}

Tissues were lysed in modified RIPA buffer (50 mM Hepes, $0.15 \mathrm{M} \mathrm{NaCl}, 2 \mathrm{mM}$ EDTA, $0.1 \%$ Nonidet $-40,0.05 \%$ sodium deoxycholate, $\mathrm{pH} 7.2)$ containing $1 \mathrm{mM} 4-(2-$ 
amnionethyl)benzenesulfonyl fluoride hydroxychloride, $0.02 \mathrm{mg} / \mathrm{ml}$ aprotonin containing $5 \%$ Triton-X. Proteins were boiled in a sample buffer and resolved using SDS-PAGE and transferred to nitrocellulose. Immunoblotting was performed using a 1:500 dilution of the appropriate primary antibody with exception of GAPDH which was used 1:1000. Blots were washed in Tris-buffered saline, $\mathrm{pH} 7.4$ plus $0.05 \%$ Triton-X, and blocked in 5\% milk, followed by incubation with the appropriate secondary antibody conjugated to horseradish peroxidase at 1:2000 dilutions (Sigma). Blots were visualized after incubation with chemiluminescence reagents (ECL, Thermo). For any reprobing, blots were stripped with Restore stripping buffer (Thermo) before being washed and restained as described above.

\section{Transthoracic Echocardiography}

Echocardiography measurements were taken at the indicated time points using previously described methods[15]. Briefly, hair was removed from the chest and abdomen, and the mice were placed on a water-jacketed, warmed table in the left lateral decubitus position for imaging. A Visualsonic Ultrasound System (Vevo 660) ultrasound machine containing a 30 $\mathrm{MHz}$ variable frequency pediatric probe with a $1 \mathrm{~cm}$ offset was used to capture echocardiograms. Standard long axis and short axis M-mode views were recorded when the mouse possessed a target heart rate between 450 and 650 beats per minute. End diastolic and end-systolic interventricular septum (IVSd, IVSs), posterior wall thickness (PWTd, PWTs) and left ventricular internal diameters (LVEDD, LVESD) were calculated and averaged from three consecutive contractions using Visual Sonics software. All animal use procedures have been reviewed and approved by the University of North Carolina's Animal Use and Care Committee.

\section{Quantification of cardiomyocyte number in serial sections}

For extrapolation of total cardiomyocyte number by sectioning and sampling, we utilized a slight modification of previously reported protocols[17, 18]. First, hearts from 4 week old mice were fixed in paraformaldehyde as above and dehydrated in methanol. The width and length of the hearts were measured after dehydration prior to embedding. After embedding, the hearts were sectioned by digital microtome, with representative $8 \mu \mathrm{m}$ sections taken every $250 \mu \mathrm{m}$ from the entire heart, with the exception of the extreme ends. The width and length were also rechecked during this time. The representative sections were then stained to distinguish cell type and morphology and random areas of the myocardium were imaged with a confocal microscope. The cells per area were counted using ImageJ software to compute a myocardial cell density. This density measurement was multiplied by the calculated heart volume (using both heart weight and heart dimensions) to quantify the number of cells per heart.

\section{RESULTS}

\subsection{Hearts from FRNK null mice exhibit sustained post-natal myocyte cycling}

We previously reported that endogenous FRNK was expressed in cardiomyocytes derived from neonatal rat and mouse myocardium[15]. Here, we further defined the duration of postnatal myocardial FRNK levels by Western blot analysis. As shown in figure 1A, FRNK levels increased rapidly in the first week of life, with the peak protein levels observed from postnatal day 7 (P7) through postnatal day 14 (P14). Low levels of FRNK were detected from postnatal day 21 to 6 weeks post-natal and FRNK was virtually undetectable thereafter. FAK expression remains relatively steady from the early postnatal period throughout adulthood in both lines of mice (Fig 1A), but myocardial FAK activity was reproducibly elevated in neonatal $\mathrm{FRNK}^{-1-}$ hearts during the second postnatal week (increased by approximately $40 \%$ at P10 compared to wt). As the timing of FRNK expression correlates with reported down-regulation of cardiomyocyte DNA synthesis and the uncoupling of 
karyo- and cyto-kinesis[6], we speculated that endogenous FRNK might be involved in these processes.

$\mathrm{FRNK}^{-/-}$mice were recently generated by homologous recombination-mediated deletion of a 1-kb fragment that included the FRNK transcriptional start site and $700 \mathrm{bp}$ of upstream $f a k$ intronic sequence. Although these mice were born in the expected Mendelian frequency and showed no gross phenotype, they have not been specifically examined for alterations in cardiac growth[16]. To evaluate a role for endogenous FRNK in the control of neonatal myocyte proliferation, we injected wild type (wt) and $\mathrm{FRNK}^{-/}$pups with $\mathrm{BrdU}$ at $\mathrm{P} 7$ and measured the extent of BrdU incorporation at P10. As shown in Figure 1B and 1C cardiomyocytes (defined by tropomyosin expression) in $\mathrm{FRNK}^{-/-}$hearts demonstrated a 2.5-fold increase in BrdU uptake compared to wt hearts, while no change in BrdU incorporation was observed in non-cardiomyocytes. Importantly, no difference in BrdU staining was observed in the small intestine of $\mathrm{FRNK}^{-/-}$and wt mice (data not shown). This finding indicates that FRNK functions to selectively limit post-natal myocardial DNA synthesis.

\subsection{Increased Histone Phosphorylation in Cardiomyocytes of FRNK ${ }^{-1-}$ Mice}

In order to examine the progression of neonatal cardiomyocytes through the cell cycle, we interrogated the timing and extent of histone phosphorylation in post-natal wt and $\mathrm{FRNK}^{-/-}$ hearts. Phosphorylation of Histone $\mathrm{H} 3$ at the serine-10 residue $\left(\mathrm{p}^{\mathrm{Ser} 10} \mathrm{H} 3\right)$ is one of the early sites of $\mathrm{H} 3$ phosphorylation and serves as a useful marker of cells that have completed $\mathrm{S}$ phase and entered G2[19]. Western blot analysis revealed a gradual decline in $\mathrm{p}^{\mathrm{Ser} 10} \mathrm{H} 3$ levels in wt hearts from P1 to P14 (Fig 2A), whereas $\mathrm{p}^{\mathrm{Ser} 10} \mathrm{H} 3$ levels were maintained at higher levels in $\mathrm{FRNK}^{-/-}$hearts. Indeed, ${ }^{\mathrm{Ser} 10} \mathrm{H} 3$ was markedly elevated in $\mathrm{P} 7$ and 14 $\mathrm{FRNK}^{-/-}$hearts compared to age-matched controls; time points when FRNK is most highly expressed in wt hearts. A significant increase in $\mathrm{p}^{\mathrm{Ser} 10} \mathrm{H} 3$ levels in $\mathrm{P} 10 \mathrm{FRNK}^{-/-}$hearts relative to littermate controls was confirmed by immunofluorescence (Fig 2B and D). Moreover, co-staining with $\mathrm{p}^{\mathrm{Ser} 10} \mathrm{H} 3$, lectin, and cardiac Troponin revealed that there were significantly more $\mathrm{p}^{\mathrm{Ser} 10} \mathrm{H} 3$ positive cardiomyocytes (Fig $2 \mathrm{C}$ and $\mathrm{E}$ ) in $\mathrm{FRNK}^{-/-}$hearts than littermate controls, while the relative number of $\mathrm{p}^{\mathrm{Ser} 10} \mathrm{H} 3$ positive non-myocytes (i.e fibroblasts, smooth muscle cells, endothelial cells; Fig. 2F) was not significantly different. As expected, given the transient nature of histone phosphorylation, $\mathrm{p}^{\text {Ser } 10} \mathrm{H} 3$ positive cardiomyocytes were less frequent than BrdU positive cells (approximately $1 \%$ vs. $10 \%$ of cardiomyocytes in P10 $\mathrm{FRNK}^{-/-}$hearts, respectively). Together these data indicate that FRNK depletion led to enhanced cardiomyocyte cell cycling from $\mathrm{S}$ phase through interphase.

\subsection{Increased Mitosis leads to Cardiomyocyte Polyploidy in FRNK ${ }^{-/-}$Hearts}

Cardiomyocytes are one of a few mammalian cells typically found in a multinucleated state in healthy adult tissue. At birth, most murine cardiomyocytes are diploid and contain a single nucleus, but these cells undergo multinucleation (due to uncoupling of mitosis from cytokinesis) between P4 and P14.[6], Thus adult mouse myocytes are typically tetraploid ( $4 \mathrm{~N}$ ) but occasionally can be found in higher levels of multiploidy (6-8N with 2 to 4 nuclei) [2]. Since we detected increased DNA synthesis in post-natal RRNK $^{-/-}$hearts, we next interrogated the extent of karyokinesis (nuclear division), by quantifying cardiomyocyte nuclei. To this end, we generated single cell suspensions from $\mathrm{FRNK}^{-/-}, \mathrm{FRNK}^{+/-}$, and WT hearts and subjected them to propidium iodide staining followed by flow cytometry (Fig $3 \mathrm{~A}$ ). As shown in figure $3 \mathrm{~B}$, at $\mathrm{P} 10$ a slight, but significant increase in $6 \mathrm{~N}$ cells was observed in $\mathrm{FRNK}^{-l-}$ hearts when compared to age-matched littermate controls and by P14, $\mathrm{FRNK}^{-1-}$ hearts contained significantly less $2 \mathrm{~N}$ cells and significantly more $4 \mathrm{~N}, 6 \mathrm{~N}$, and $8 \mathrm{~N}$ cells relative to controls (Fig 3C). This change resulted in an approximate $10 \%$ increase in 
average DNA content per cell in P14 $\mathrm{FRNK}^{-/-}$hearts relative to age-matched controls. To establish that the increased polyploidy at P14 was actually due to higher DNA/cell content in cardiomyocytes, we co-stained cells from P14 $\mathrm{FRNK}^{-l-}$ and $\mathrm{FRNK}^{-/+}$littermate hearts for $\mathrm{N}$-cadherin prior to subjecting them to flow cytometry. $\mathrm{N}$-cadherin is a transmembrane adhesion protein present in several cell types, but within the myocardium it is expressed nearly exclusively in cardiomyocytes[20]. As shown in figure $3 \mathrm{E}, \mathrm{F}$, the $4 \mathrm{~N}$ peak was predominantly composed of $\mathrm{N}$-cadherin expressing cells (approximately $80 \%$ were $\mathrm{N}$ cadherin positive) whereas the $6 \mathrm{~N}$ and $8 \mathrm{~N}$ peaks were exclusively found in the $\mathrm{N}$-Cadherin positive population; indicating that these peaks largely represent bi-, tri- and tetra-nucleated cardiomyocytes. Notably, at this timepoint while more 4, 6 and $8 \mathrm{~N}$-containing cardiomyocytes were evident in the $\mathrm{FRNK}^{-/-}$hearts, there was no indication of a greater proportion of cardiomyocytes. Collectively, these results indicate FRNK depletion led to increased DNA synthesis which culminated in elevated karyokinesis between P10 and P14.

Interestingly, the increased ploidy observed in post-natal $\mathrm{FRNK}^{-/-}$hearts was transient and by P21, the extent of multi-nucleation was similar between FRNK null and FRNK containing hearts (Fig 3D). To determine whether the normalization of polyploidy in the P21 $\mathrm{FRNK}^{-/}$hearts might have resulted from subsequent cytokinesis, we evaluated the expression and locale of Aurora B, a protein essential for the progression from mitotic entry through cytokinesis[21]. Consistent with selective expression of Aurora B in cells undergoing mitotic division, we found high levels of cardiomyocyte-specific Aurora-B staining in embryonic mouse hearts (Fig 4A) and lower levels in postnatal hearts (Fig 4B). However, a significant increase in Aurora-B positive cardiomyocytes were observed in both P14 and P20 FRNK ${ }^{-/}$hearts compared to age-matched controls (Fig 4B-D, and G) in the absence of elevated DNA synthesis (Fig 4G left). Moreover, when scored for active or recent cytokinetic events (as defined by the presence of a mitotic spindle- a hallmark of cytokinesis or 2 closely apposed Aurora B positive cardiomyocyte nuclei), $3.5 \%$ of $\mathrm{FRNK}^{-/}$cardiomyocytes scored positive in comparison to approximately $1 \%$ of wt cardiomyocytes at P20 (Fig 4H). While we found a similar elevation in Aurora B staining within FRNK $^{-1-}$ hearts at P14, apparent cytokinesis was more rarely observed at this timepoint (data not shown). It is important to note that the increased cell cycling associated with FRNK null hearts in the early neonatal window subsided by this time point. Thus, when combined with our flow analysis, these findings support the possibility that elevated cardiomyocyte polyploidy in FRNK null hearts was resolved by subsequent cytokinesis.

\subsection{Increased Left Ventricular Mass in FRNK ${ }^{-1-}$ Mice}

We next subjected neonatal wt and $\mathrm{FRNK}^{-/}$mice to serial echocardiograms to assess the effect of FRNK depletion on cardiac growth. While left ventricular thickness was not different at week 1 , both the interventricular septum and posterior wall were thicker in $\mathrm{FRNK}^{-1-}$ mice by 4 weeks out to 6 weeks of age (the termination of the experiment; Fig 5A, $\mathrm{B}$ and Supplemental Table 1). The total ventricular mass, calculated either by echocardiography or direct weight measurements, was also significantly increased in $\mathrm{FRNK}^{-/-}$hearts compared to age-matched controls. (Fig 5C) This finding of significantly increased ventricular mass/body mass and ventricular mass/tibia length measurements was corroborated in a second, larger cohort of age- and sex-matched mice sacrificed at 6 weeks of age (Supplemental Table 2).

In order to determine whether the increased LV wall thickness and mass resulted from myocyte hypertrophy, hyperplasia or both, we determined the cross-sectional cardiomyocyte area and the number of cardiomyocytes in $\mathrm{FRNK}^{-1-}$ and wt hearts per $\mathrm{mm}^{2}$. As shown in supplemental figure 1, there was no significant difference in cardiomyocyte size in 6 week old wt and $\mathrm{FRNK}^{-/-}$hearts. We also examined single cell suspensions of cardiomyocytes and did not observe a difference in the size of isolated $\mathrm{FRNK}^{-/-}$myocytes compared to wt 
(Supplemental Figure 2). Likewise, there was no significant difference in the total number of myocytes per unit area of muscle nor in the ratio of myocyte area to interstitial area, indicating that the observed increase in heart size did not result from an increase in interstitial or vascular cells. We next strove to quantify total cardiomyocyte number in 4 week post-natal wt and $\mathrm{FRNK}^{-/-}$hearts. To this end, hearts from 4 week old mice were processed and cardiomyocytes were quantified by 2 distinct methods: completion flow cytometry and direct counting of random serial sections as reported elsewhere[16, 17]. Both methodologies revealed a similar increase in the absolute number of cardiomyocytes in $\mathrm{FRNK}^{-/-}$hearts. As shown in Figure 5D, using flow cytometry we calculated a total of $3.9 \times 10^{6}$ myocytes per wt ventricle vs. $5.0 \times 10^{6}$ in $\mathrm{FRNK}^{-1-}$; representing a $28 \%$ increase in cardiomyocytes/ventricle. Using random sampling we calculated $1.7 \times 10^{6}$ myocytes in wt ventricle vs. $1.9 \times 10^{6}$ in $\mathrm{FRNK}^{-/-}$, resulting in a $12 \%$ increase (data not shown).

Collectively, these findings indicate that lack of FRNK expression prolonged cardiomyocyte cell cycle progression in postnatal hearts which culminated in increased total numbers of cardiomyocytes and larger hearts.

\section{DISCUSSION}

FRNK expression in the developing myocardium is restricted to a critical post-natal window during which cardiomyocytes withdraw from the proliferative cell cycle and undergo binucleation. We previously reported that mis-expression of FRNK in the embryonic heart led to pre-term lethality associated with reduced cardiomyocyte proliferation and ventricular non-compaction[15]. Herein, we provide strong evidence that endogenous FRNK contributes to post-mitotic arrest, as $\mathrm{FRNK}^{-/-}$hearts exhibited prolonged neonatal cell cycling and contained larger hearts with more cardiomyocytes than age- and littermate matched controls.

Based on our previously published report of FRNK expression in the neonatal myocardium[15], in a pattern inversely correlated with cardiomyocyte proliferation[6], we speculated that FRNK functioned to promote quiescence in this matrix and growth factorrich environment that is positioned to promote high levels of FAK activity. Indeed, hearts from $\mathrm{P} 10 \mathrm{FRNK}^{-/-}$mice showed increased numbers of BrdU and $\mathrm{p}^{\mathrm{Ser} 10} \mathrm{H} 3$ positive cardiomyocyte nuclei when compared to age-matched controls, consistent with delayed cessation of myocyte cell cycling. As expected, the prolonged cell cycling observed in $\mathrm{FRNK}^{-/-}$hearts was transient, and subsided at about the time when FRNK levels had markedly dropped in wt hearts (P21). This finding implies that induction of FRNK aids in restricting post-natal S-phase entry, but that additional mechanisms also regulate cell cycle withdraw. It is important to note that endogenous FRNK levels subside at time points that are concurrent with changes in myocardial extracellular matrix deposition that involve a switch from the growth promoting matrix, fibronectin (a strong FAK activator), to the growth limiting matrix collagen (a weak FAK activator)[8], although it is formally possible that FRNK acts to control cardiomyocyte proliferation by a FAK-independent mechanism. As well, marked changes in cell cycle regulators are observed in this late post-natal window that certainly contribute to terminal differentiation[22]. Nonetheless, FRNK appears to be necessary for the rapid transition to post-natal cardiomyocyte quiescence as mitotic activity was significantly prolonged in $\mathrm{FRNK}^{-/-}$hearts.

Several studies indicate that myocyte bi-nucleation results from a block between karyo- and cytokinesis that occurs within the first two weeks of postnatal murine heart development [6, 23]. In accordance with these findings, we showed that elevated DNA synthesis in the FRNK null hearts was coupled with increased DNA content and multi-nucleation by P14, indicative of DNA replication without cell division. These data indicate that endogenous FRNK may serve an important role in limiting excess ploidy during the transition stage from 
mono- to bi-nucleated cardiomyocytes. While our data indicated an extra nucleus for every 10 cardiomyocytes on average in FRNK null hearts, we may have underestimated the total effect of the absence of FRNK given the reports of FAK regulation of N-Cadherin levels[24, 25]. Interestingly, the extent of multi-nucleation in $\mathrm{FRNK}^{--}$hearts subsided over time and levels of tri- and tetra-nucleated myocytes were not significantly different from wt mice by P21. Thus, cardiomyocytes may have an intrinsic homeostatic mechanism that governs nuclear number. In fact a precedent exists for this phenomenon. For example, transient increases in multi-nucleation have been reported to occur in patients following an acute ischemic insult[3]. Moreover, Wohlschlaeger et al. reported that the polyploidy associated with failing human hearts was reversed upon mechanical unloading by left ventricular assist device implantation[26]. As well, studies in a mouse model of sepsis indicated that cardiomyocyte polyploidy and multi-nucleation increased during the acute phase of severe infection, but normalized when the infection subsided[27]. While the fate of the multiploid cells was not fully evaluated in these settings, the study by Wohlschlager et. al. reported that there was no evidence of apoptosis, indicating that these cells might survive and divide, consistent with the ultimate outcome of improved tissue health.

The following findings support the likelihood that the transient multi-nucleation observed in FRNK depleted cardiomyocytes was resolved by cell division: 1) a reduction in tri- and tetra-nucleated cardiomyocytes and a concomitant increase in bi-nucleated cells occurred in $\mathrm{FRNK}^{-/-}$hearts between 14 and 21 days post-natal 2) Aurora B-labeled central spindles (a hallmark of cytokinesis) were observed in tetra-nucleated P20 $\mathrm{FRNK}^{-/-}$but not wt cardiomyocytes 3) no evidence of increased apoptotic cardiomyocytes was observed in postnatal FRNK null hearts relative to age-matched controls (as assessed by TUNEL staining; data not shown) and 4) hearts from FRNK null mice developed ventricular enlargement that persisted at least until young adulthood and these hearts contained a significantly increased population of myocytes. Thus, we speculate that deletion of FRNK releases a brake on DNA synthesis that is followed by increased karyokinesis and eventual cytokinesis. We believe that the absence of FRNK induces a delay in both the initiation as well as the duration in cytokinesis, reflected by the observation that Aurora B levels were elevated prior to any evidence of cell division. The delay in cytokinesis may be a result of failed attempts of appropriate contractile ring formation that is known to occur in post-natal cardiomyocytes[28]. Alternatively, since it is unlikely that an appropriately polarized contractile ring could form in tri-nucleated cells, the lag could reflect the additional time required for the 2 rounds of DNA synthesis that are necessary to form a tetra-nucleated cell with cytokinetic potential. We surmise that the increased numbers of multi-ploid cells undergoing division in $\mathrm{P} 20 \mathrm{FRNK}^{-/-}$hearts might be a simple reflection of the fact that nearly 2-fold more tetra-nucleated cells are present in P14 $\mathrm{FRNK}^{-1-}$ compared to agematched wt hearts. However, it has been suggested that additional cell cycle checkpoints must be circumvented for cytokinesis to occur in multi-ploid cells[29]. Thus an equally plausible explanation for our findings is that FRNK might regulate both G1/S- and G2/Mcheckpoint dependent cell cycle withdrawal. While the relative increase in HW observed in $\mathrm{FRNK}^{-/-}$mice was modest, likely due to the fact that $\mathrm{FRNK}^{-/-}$cardiomyocytes do not escape eventual cell cycle withdrawal, it was similar to the extent of hyperplastic growth induced cardiac expression of the potent cell cycle regulator, $\mathrm{cMyc}[30]$.

Our studies provide clear evidence that FRNK depletion leads to elevated DNA synthesis in post-natal hearts that eventually leads to myocyte hyperplasia and cardiac augmentation. In this regard, FRNK appears to govern tight coupling between DNA synthesis, karyokinesis, and cytokinesis in the neonatal heart. Although mitosis is a rare event in the adult myocardium[1], several high profile papers have revealed that myocyte proliferation does occur following myocardial infarction in both rodent and human hearts[31][32], indicating that appropriately controlled regeneration of myocytes could be a mechanism to replenish 
lost muscle after a heart attack. Whether FRNK is re-expressed during cardiac disease progression and whether it functions in a conserved manner to regulate ploidy in the adult myocardium are important questions for future interrogation.

\section{Supplementary Material}

Refer to Web version on PubMed Central for supplementary material.

\section{References}

1. Kajstura J, Urbanek K, Perl S, Hosoda T, Zheng H, Ogorek B, et al. Cardiomyogenesis in the Adult Human Heart. Circ Res. 2010; 107:305-15. [PubMed: 20522802]

2. Walsh S, Pontén A, Fleischmann BK, Jovinge S. Cardiomyocyte cell cycle control and growth estimation in vivo - an analysis based on cardiomyocyte nuclei. Cardiovascular Research. 2010; 86:365-73. [PubMed: 20071355]

3. Meckert PC, Rivello HG, Vigliano C, González P, Favaloro R, Laguens R. Endomitosis and polyploidization of myocardial cells in the periphery of human acute myocardial infarction. Cardiovascular Research. 2005; 67:116-23. [PubMed: 15949475]

4. Porrello ER, Mahmoud AI, Simpson E, Hill JA, Richardson JA, Olson EN, et al. Transient Regenerative Potential of the Neonatal Mouse Heart. Science. 2011; 331:1078-80. [PubMed: 21350179]

5. Wang J, Panáková D, Kikuchi K, Holdway JE, Gemberling M, Burris JS, et al. The regenerative capacity of zebrafish reverses cardiac failure caused by genetic cardiomyocyte depletion. Development. 2011; 138:3421-30. [PubMed: 21752928]

6. Soonpaa MH, Kim KK, Pajak L, Franklin M, Field LJ. Cardiomyocyte DNA synthesis and binucleation during murine development. American Journal of Physiology - Heart and Circulatory Physiology. 1996; 271:H2183-H9.

7. Bicknell KA, Coxon CH, Brooks G. Can the cardiomyocyte cell cycle be reprogrammed? Journal of Molecular and Cellular Cardiology. 2007; 42:706-21. [PubMed: 17362983]

8. Ieda M, Tsuchihashi T, Ivey KN, Ross RS, Hong T-T, Shaw RM, et al. Cardiac Fibroblasts Regulate Myocardial Proliferation through $\beta 1$ Integrin Signaling. Developmental cell. 2009; 16:233-44. [PubMed: 19217425]

9. Hakim ZS, DiMichele LA, Doherty JT, Homeister JW, Beggs HE, Reichardt LF, et al. Conditional Deletion of Focal Adhesion Kinase Leads to Defects in Ventricular Septation and Outflow Tract Alignment. Mol Cell Biol. 2007; 27:5352-64. [PubMed: 17526730]

10. Peng X, Wu X, Druso JE, Wei H, Park AY-J, Kraus MS, et al. Cardiac developmental defects and eccentric right ventricular hypertrophy in cardiomyocyte focal adhesion kinase (FAK) conditional knockout mice. Proceedings of the National Academy of Sciences. 2008; 105:6638-43.

11. Parsons JT, Slack-Davis J, Tilghman R, Roberts WG. Focal Adhesion Kinase: Targeting Adhesion Signaling Pathways for Therapeutic Intervention. Clinical Cancer Research. 2008; 14:627-32. [PubMed: 18245520]

12. Richardson A, Parsons JT. A mechanism for regulation of the adhesion-associated protein tyrosine kinase pp125FAK. Nature. 1996; 380:538-40. [PubMed: 8606775]

13. Katz B-Z, Miyamoto S, Teramoto H, Zohar M, Krylov D, Vinson C, et al. Direct transmembrane clustering and cytoplasmic dimerization of focal adhesion kinase initiates its tyrosine phosphorylation. Biochimica et Biophysica Acta (BBA) - Molecular Cell Research. 2002; 1592:141-52.

14. Heidkamp MC, Bayer AL, Kalina JA, Eble DM, Samarel AM. GFP-FRNK Disrupts Focal Adhesions and Induces Anoikis in Neonatal Rat Ventricular Myocytes. Circulation Research. 2002; 90:1282-9. [PubMed: 12089066]

15. DiMichele LA, Hakim ZS, Sayers RL, Rojas M, Schwartz RJ, Mack CP, et al. Transient Expression of FRNK Reveals Stage-Specific Requirement for Focal Adhesion Kinase Activity in Cardiac Growth. Circ Res. 2009; 104:1201-8. [PubMed: 19372463] 
16. Hayasaka H, Martin KH, Hershey ED, Parsons JT. Disruption of FRNK expression by gene targeting of the intronic promoter within the focal adhesion kinase gene. Journal of Cellular Biochemistry. 2007; 102:947-54. [PubMed: 17440961]

17. Anversa P, Palackal T, Sonnenblick E, Olivetti G, Meggs L, Capasso J. Myocyte cell loss and myocyte cellular hyperplasia in the hypertrophied aging rat heart. Circulation Research. 1990; 67:871-85. [PubMed: 2145091]

18. Brüel A, Nyengaard JR. Design-based stereological estimation of the total number of cardiac myocytes in histological sections. Basic Research in Cardiology. 2005; 100:311-9. [PubMed: 15795797]

19. Cheung P, Allis CD, Sassone-Corsi P. Signaling to Chromatin through Histone Modifications. Cell. 2000; 103:263-71. [PubMed: 11057899]

20. Li J, Patel VV, Kostetskii I, Xiong Y, Chu AF, Jacobson JT, et al. Cardiac-Specific Loss of NCadherin Leads to Alteration in Connexins With Conduction Slowing and Arrhythmogenesis. Circ Res. 2005; 97:474-81. [PubMed: 16100040]

21. Katayama H, Sen S. Aurora kinase inhibitors as anticancer molecules. Biochimica et Biophysica Acta (BBA) - Gene Regulatory Mechanisms. 2010; 1799:829-39.

22. Poolman RA, Brooks G. Expressions and Activities of Cell Cycle Regulatory Molecules During the Transition from Myocyte Hyperplasia to Hypertrophy. Journal of Molecular and Cellular Cardiology. 1998; 30:2121-35. [PubMed: 9799664]

23. Liu Z, Yue S, Chen X, Kubin T, Braun T. Regulation of Cardiomyocyte Polyploidy and Multinucleation by CyclinG1. Circulation Research. 2010; 106:1498-506. [PubMed: 20360255]

24. Playford MP, Vadali K, Cai X, Burridge K, Schaller MD. Focal Adhesion Kinase regulates cellcell contact formation in epithelial cells via modulation of Rho. Experimental Cell Research. 2008; 314:3187-97. [PubMed: 18773890]

25. Yamada K, Green KG, Samarel AM, Saffitz JE. Distinct Pathways Regulate Expression of Cardiac Electrical and Mechanical Junction Proteins in Response to Stretch. Circulation Research. 2005; 97:346-53. [PubMed: 16037569]

26. Wohlschlaeger J, Levkau B, Brockhoff G, Schmitz KJ, von Winterfeld M, Takeda A, et al. Hemodynamic Support by Left Ventricular Assist Devices Reduces Cardiomyocyte DNA Content in the Failing Human Heart. Circulation. 2010; 121:989-96. [PubMed: 20159834]

27. Anatskaya OV, Sidorenko NV, Beyer TV, Vinogradov AE. Neonatal cardiomyocyte ploidy reveals critical windows of heart development. International journal of cardiology. 2010; 141:81-91. [PubMed: 19138803]

28. Engel FB, Schebesta M, Keating MT. Anillin localization defect in cardiomyocyte binucleation. Journal of Molecular and Cellular Cardiology. 2006; 41:601-12. [PubMed: 16889791]

29. Pasumarthi KBS, Field LJ. Cardiomyocyte Cell Cycle Regulation. Circulation Research. 2002; 90:1044-54. [PubMed: 12039793]

30. Jackson T, Allard MF, Sreenan CM, Doss LK, Bishop SP, Swain JL. The c-myc proto-oncogene regulates cardiac development in transgenic mice. Mol Cell Biol. 1990; 10:3709-16. [PubMed: 1694017]

31. Beltrami AP, Urbanek K, Kajstura J, Yan S-M, Finato N, Bussani R, et al. Evidence That Human Cardiac Myocytes Divide after Myocardial Infarction. New England Journal of Medicine. 2001; 344:1750-7. [PubMed: 11396441]

32. Maximilian Buja L, Vela D. Cardiomyocyte death and renewal in the normal and diseased heart. Cardiovascular Pathology. 2008; 17:349-74. [PubMed: 18402842] 


\section{Highlights}

- Focal adhesion kinase-related non-kinase (FRNK) limits perinatal cell cycling

- Myocytes from FRNK-null mice exhibit sustained DNA synthesis and transient polyploidy

- FRNK-null hearts exhibit hyperplastic growth 

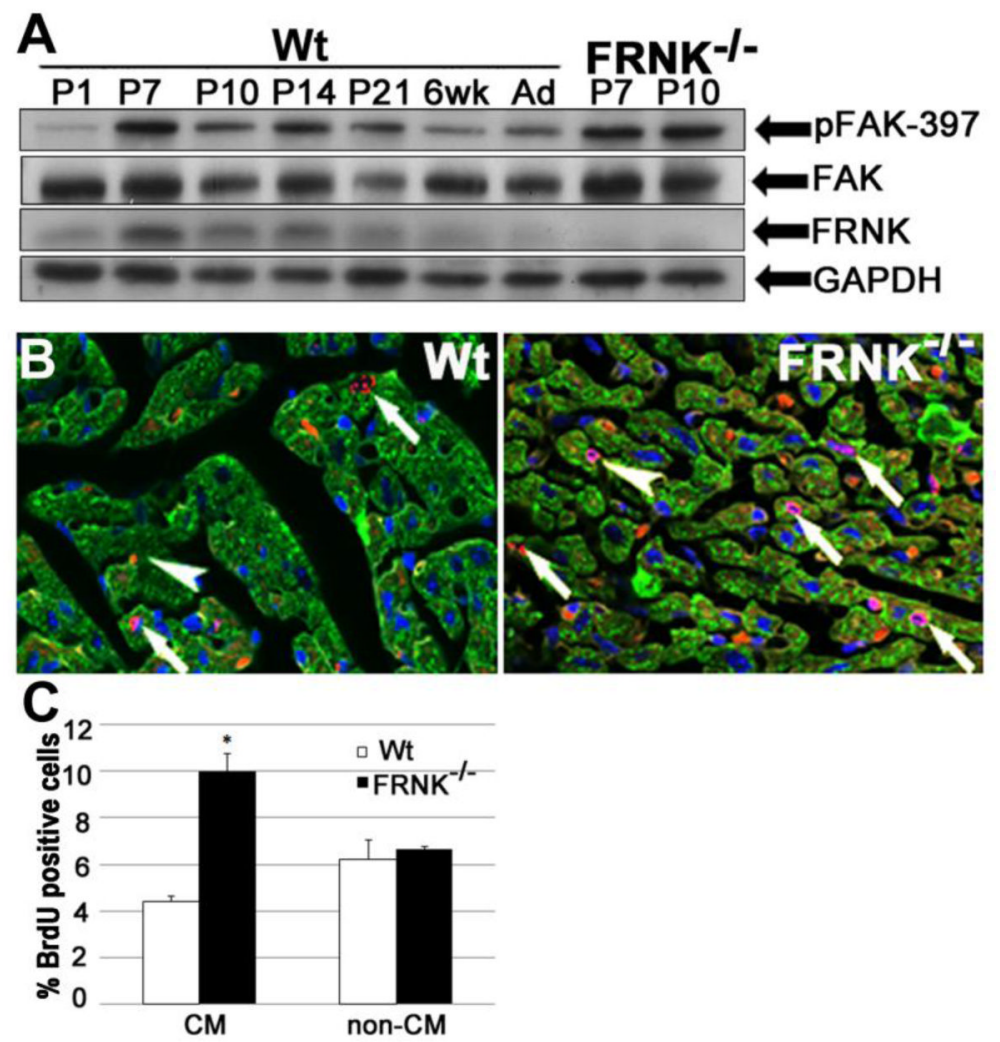

Figure 1. Neonatal FRNK null hearts exhibit elevated levels of DNA synthesis A. Western blot showing temporal expression of FRNK, FAK, and phospho-397 FAK (pFAK-397) in wild-type mice (Wt) from postnatal day 1 (P1) to 6 weeks (6 wk) and adult (Ad), with GAPDH shown as loading control. FRNK expression peaks between P7 and P10 while FAK expression remains stable. Far right lanes reveal expected absence of FRNK protein as well as increased pFAK-397 in P7 and P10 FRNK $^{-/-}$hearts. FAK activity was elevated most consistently at P10. B. BrdU incorporation in P10 Wt and $\mathrm{FRNK}^{-/-}$ventricles was detected by immunofluorescence. BrdU+ cells are red, while DAPI (blue) and cardiac tropomyosin (green) are shown to distinguish BrdU+ cardiomyocyte (arrows) from noncardiomyocytes (arrowheads). C. Quantification of \% of BrdU-positive cardiomyocytes (CM) and non-cardiomyocytes (non-CM) in wt and $\mathrm{FRNK}^{-/-}$hearts (means \pm SEM; $\mathrm{n} 42$ $* P<0.01$, magnification $=600 \times)$. 

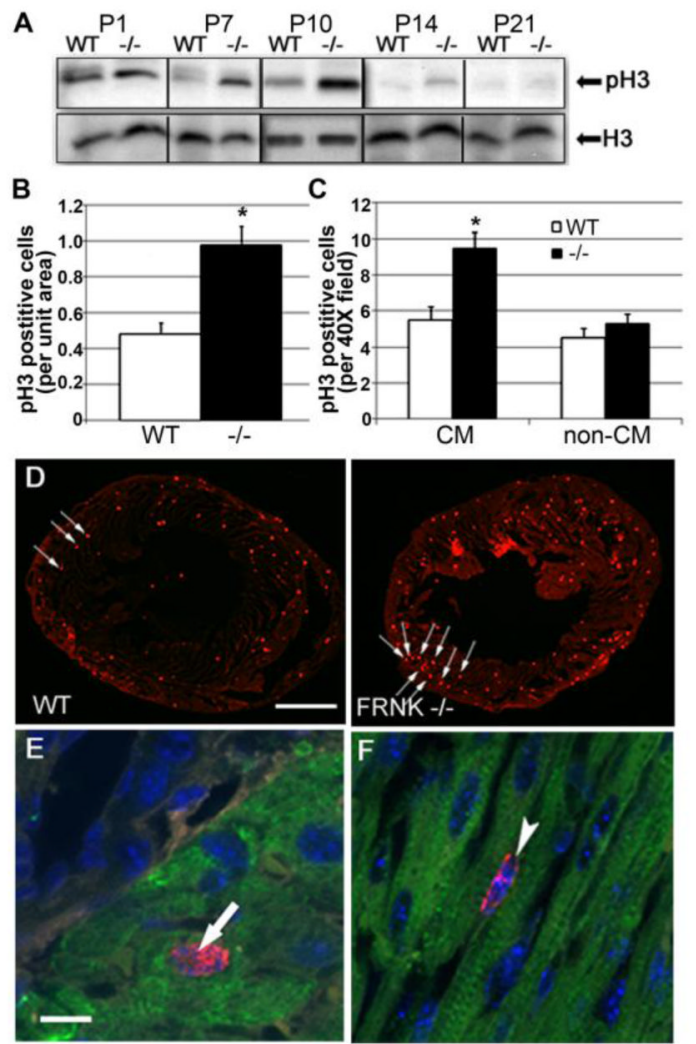

Figure 2. Depletion of FRNK leads to prolonged postnatal myocyte mitosis

A. Western blot analysis showing relative levels of $\mathrm{p}^{\mathrm{Ser} 10}$ histone $\mathrm{H} 3(\mathrm{pH} 3)$ and total histone $\mathrm{H} 3$ (H3) from postnatal $\mathrm{Wt}$ and $\mathrm{FRNK}^{-/-}$hearts (collected from 1 day old (P1) to 21 day old mice (P21). B-F Immunohistochemistry for pH3 in P10 wt and $\mathrm{FRNK}^{-/-}$hearts. (B)

Quantification of total $\mathrm{pH} 3$ positive cells/unit area $(\mathrm{n}=6,7 . *-\mathrm{p} \unlhd 0.05)$. (C) Quantification of $\mathrm{pH} 3$ positive cardiomyocytes (CM) and non-cardiomyocytes (non-CM) as assessed by costaining with cardiac troponin I (n=6,6.*-p $₫ 0.05)$. (D) Low power immunofluorescent staining of heart sections for $\mathrm{pH} 3$ (red), $\mathrm{pH} 3$ positive nuclei indicate by arrows. Scale bar $=$ $1 \mathrm{~mm}$. E,F. High power immunofluorescent staining showing $\mathrm{pH} 3$ positive cardiomyocyte (arrow, panel E) and interstitial cell (arrowhead, panel $\mathbf{F})$. Red $=$ pH3. Blue= DAPI. Green= Troponin I. Scale bar $=10 \mu \mathrm{m}$. 

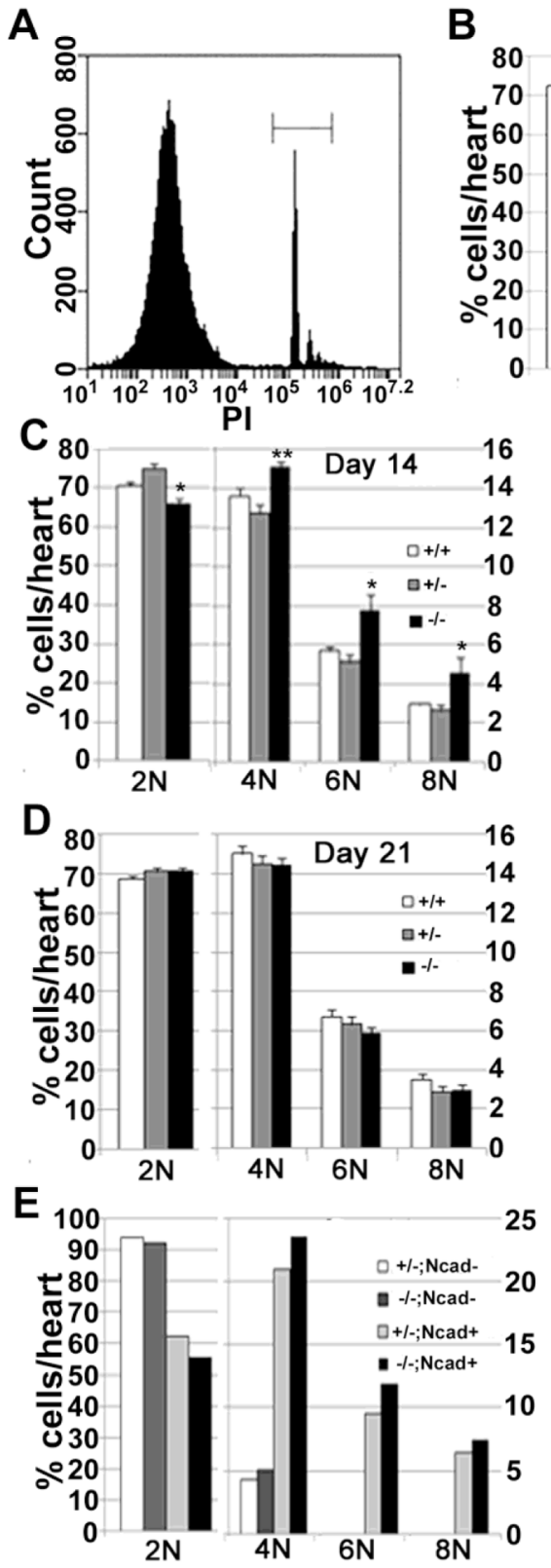

B

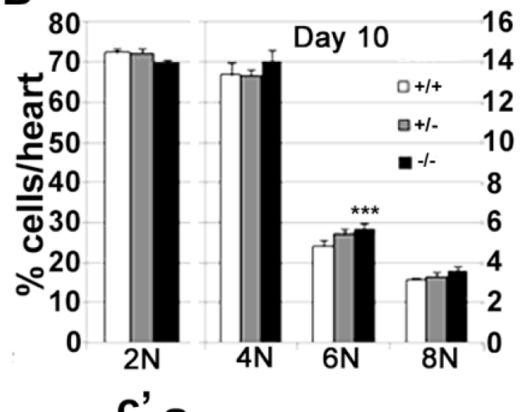

c'。

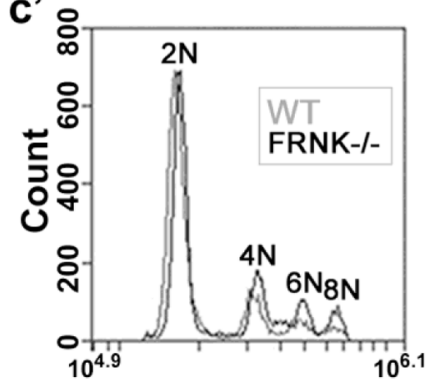

$\mathbf{F}$
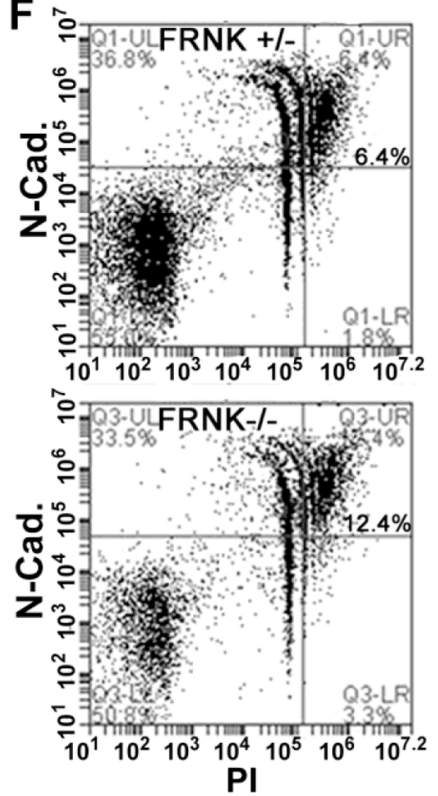

Figure 3. FRNK depletion leads to a transient elevation in cardiomyocyte multi-nucleation A. Representative propidium iodide (PI) staining of cardiac cells showing gating on PI positive cells (denoted by bracket). B-D. Quantification of relative numbers of $2 \mathrm{~N}, 4 \mathrm{~N}, 6 \mathrm{~N}$ and $8 \mathrm{~N}$ positive cells from wild type (+/+), $\mathrm{FRNK}^{+/-}(+/-)$, and $\mathrm{FRNK}^{-/-}(-/-)$hearts at postnatal day 10,14 and 21. $\mathbf{c}^{\prime}$ shows a representative histogram showing relative DNA content in cells from $\mathrm{FRNK}^{-/}$(black) and WT (grey) P14 hearts. (Flow experiments represent at least 2 experiments per data point, $\mathrm{n}=\mathrm{P} 10-6,9,8$; $\mathrm{P} 14-5,9,4$; $\mathrm{P} 21-6,9,8$ for wt, FRNK $^{+/-}$, and $\mathrm{FRNK}^{-/-}$respectively. *-- $\mathrm{p} \unlhd 0.05$ for $\mathrm{FRNK}^{-/-}$vs. both FRNK ${ }^{+/-}$and WT, $* *$ - $\mathrm{p} ₫ 0.05$ only vs. FRNK ${ }^{+/-}$, ***- $\mathrm{p} \unlhd 0.05$ only vs. WT. E-F. DNA content of N-cadherin positive $(\mathrm{Ncad}+)$ and negative $(\mathrm{Ncad}-)$ cells with a scatter plot $(\mathbf{F})$ showing increased DNA content in $\mathrm{Ncad}+$ cells from $\mathrm{FRNK}^{-/-}$hearts in upper right quadrant. Values represent pooled samples from 6-7 hearts. 


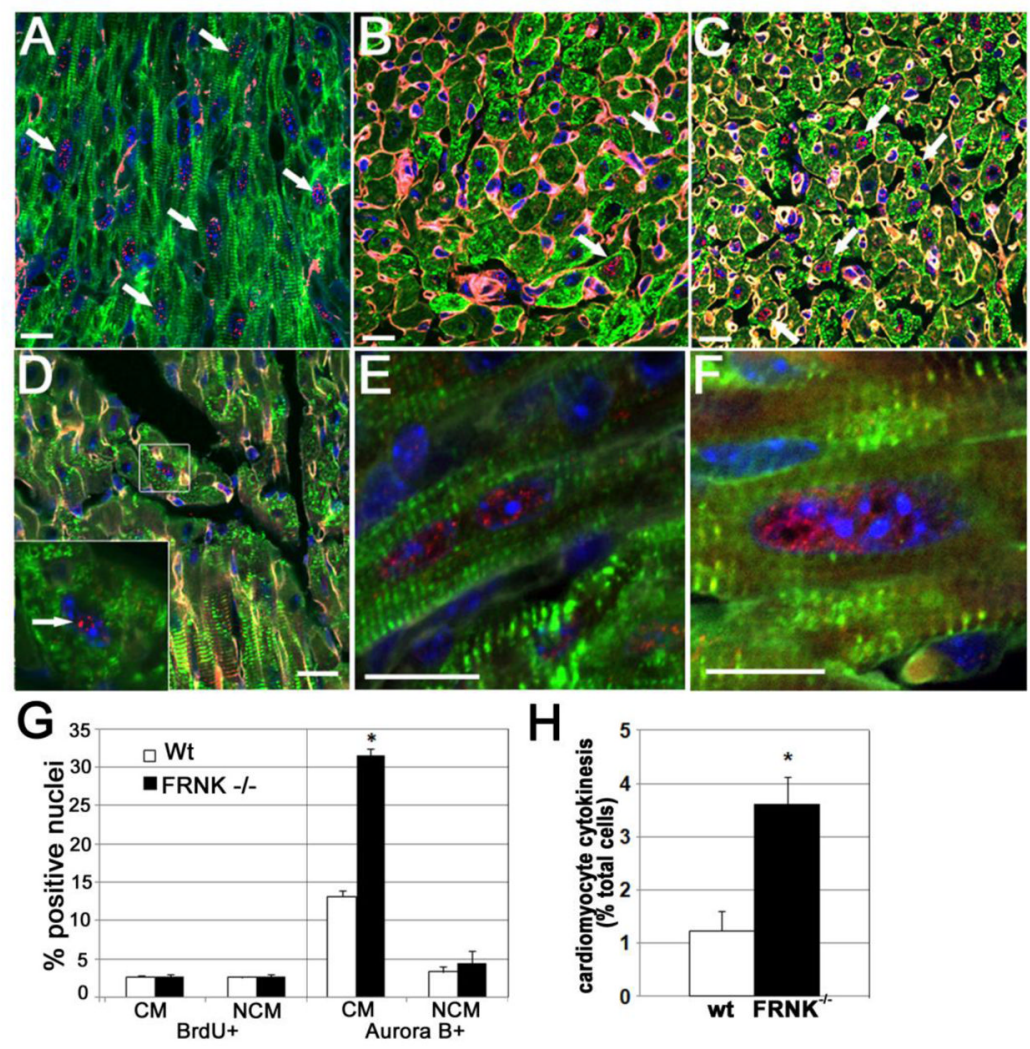

Figure 4. Nuclear Aurora B signal was maintained in postnatal RRNK $^{-/-}$hearts

A. Embryonic wt heart showing nearly ubiquitous Aurora B expression in cardiomyocyte nuclei (arrows). B and C. P 14 wt (B) hearts show diminished Aurora B expression relative to embryonic hearts and P14 FRNK $^{-/}$hearts $(\mathbf{C})$ D-H. Aurora B expression persists in P20 FRNK $^{-/-}$cardiomyocytes (particularly in those containing enlarged nuclei). Note Aurora B location along central axis indicative of karyokinesis (left panel). G. Quantification showing increase in Aurora-B positive cells are not accounted for by prolonged proliferation: percentages of BrdU positive (left) and Aurora-B positive (right) cardiomyocytes (CM) and non-cardiomyocytes (NCM) at P20 H. Percentages of Aurora-B positive myocytes at P20 that appeared to be in active or recent cytokinesis based on the pattern of Aurora-B staining. $($ Green $=$ tropomyosin, Blue $=$ DAPI, Red $=$ Aurora B, White $=$ WGA lectin; $n=4 . *-p ~ \$ 0.01)$. Scale bar $=100$ microns. 

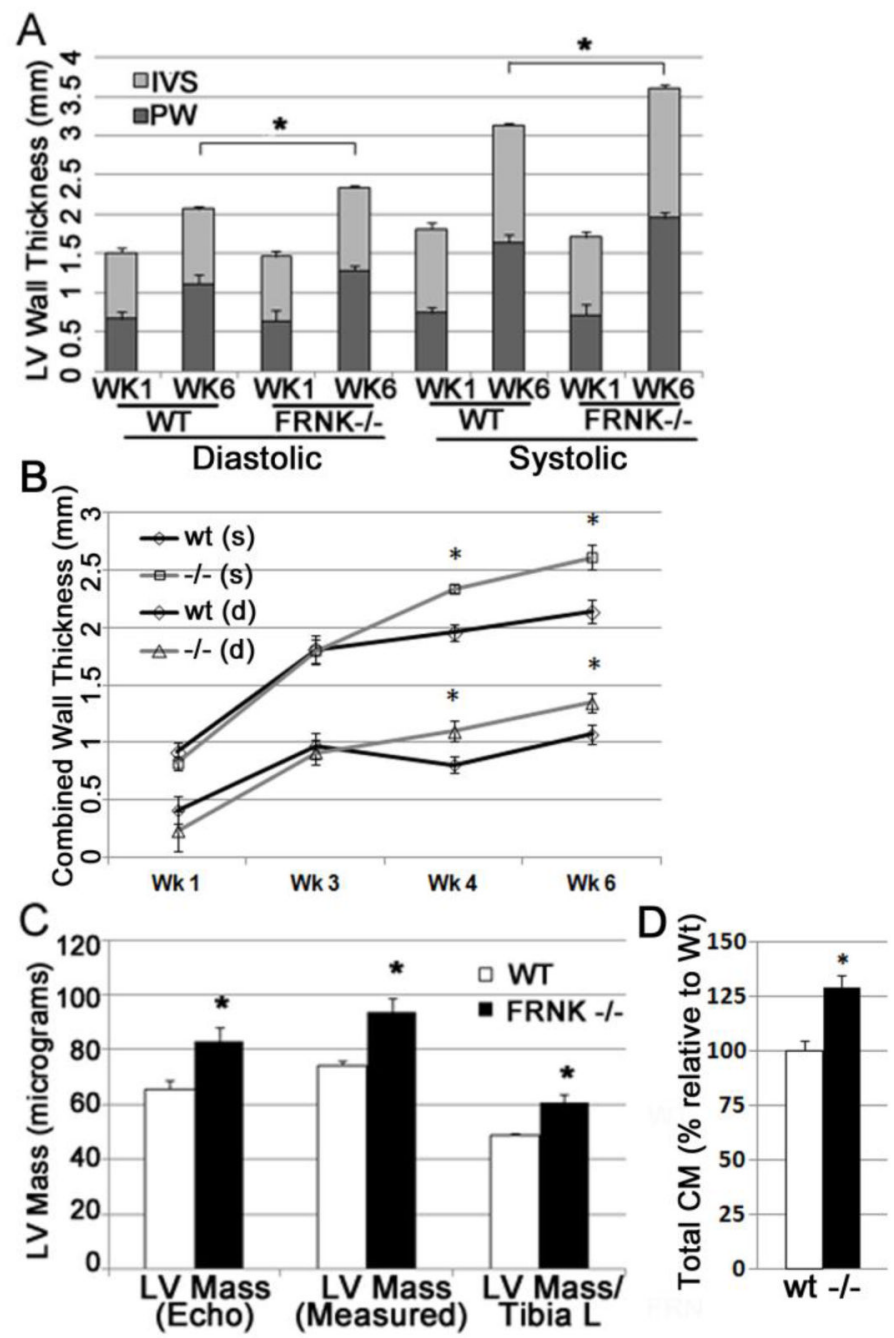

Figure 5. Postnatal $\mathrm{FRNK}^{-/-}$hearts exhibit ventricular augmentation

A. Echocardiographic measurements of left ventricle (LV) interventricular septum (IVS), and posterior wall (PW) at 1 and 6 weeks in wt and $\mathrm{FRNK}^{-/-}$mice $(\mathrm{n}=7$ and 5). B. Time course of combined (IVS + PW) systolic and diastolic left ventricular wall thickness from week 1 through week 6. C. LV mass approximated by echo, compared to direct measurements of heart mass and heart mass adjusted for tibia length. D. Total cardiomyocytes per heart in 4 week wt and $\mathrm{FRNK}^{-/-}$hearts quantified by flow cytometry $(\mathrm{n}=6 . *$-p $\unlhd(01)$. 